\title{
Theoretical solutions to three-dimensional asymmetrical shock/shock interaction
}

\author{
XIANG GaoXiang ${ }^{1}$, WANG Chun ${ }^{1 *}$, HU ZongMin ${ }^{1}$, LI XuDong ${ }^{2} \&$ JIANG ZongLin ${ }^{1}$ \\ ${ }^{1}$ State Key Laboratory of High Temperature Gas Dynamics (LHD), Institute of Mechanics, Chinese Academy of Sciences, Beijing 100190, \\ China; \\ ${ }^{2}$ Beijing Institute of Aerospace Long March Vehicle, Beijing 100076, China
}

Received December 8, 2015; accepted March 3, 2016; published online March 29, 2016

\begin{abstract}
This paper explores theoretical solutions to the three-dimensional (3D) shock/shock interaction induced by 3D asymmetrical intersecting compression wedges in supersonic inviscid flows. For Mach interactions, an analytical method known as spatial-dimension reduction, which transforms the problem of 3D steady shock/shock interaction into a two-dimensional (2D) pseudo-steady problem on cross sections, is used to obtain the solutions in the vicinity of the Mach stem. The theoretical solutions include the pressure, temperature, density, Mach number behind the Mach stem, and total pressure recovery coefficient. Numerical simulations are performed to validate the theoretical results. Here, the NND scheme is employed by solving 3D inviscid Euler equations, and good agreements are obtained. The asymmetry of the flow characteristics induced by the wedge angle and sweep angle are thoroughly discussed.
\end{abstract}

3D asymmetrical shock/shock interaction, spatial-dimension reduction, Mach reflection, flow field, theoretical analysis

Citation: Xiang G X, Wang C, Hu Z M, et al. Theoretical solutions to three-dimensional asymmetrical shock/shock interaction. Sci China Tech Sci, 2016, 59: 1208-1216, doi: 10.1007/s11431-016-6036-Z

\section{Introduction}

In aerospace engineering, supersonic corner flow is an important issue owing to its existence in wing roots, wing-fin junctions, air intakes, etc. (see Figure 1). It produces complex three-dimensional (3D) wave configurations dominated by shock/shock interactions and induces a high heat flux and large total pressure loss.

The shock/shock interaction induced by two intersecting wedges has been studied, and remarkable progress has been made via several experimental and computational investigations [1-17]. Charwat, Cresci, Watson, and West et al. [1-4] conducted the earliest experimental research on the distribution of the surface pressure and the wave structure in the

\footnotetext{
*Corresponding author (email: wangchun@imech.ac.cn)
}

corner region for both symmetrical corner and asymmetrical models. Cresci, Stainback, Korkegi, and Venkateswaran et al. [2,5-7] performed experimental studies on aerodynamic heating in the vicinity of corners. With the rapid development of computer technology and computational fluid dynamics, numerical methods are used to investigate the corner flows conveniently and economically [8-17]. Rubin, Kutler, Shang, and Marconi et al. [8-11] did the earliest numerical studies on characteristics of corner flows using a low-order numerical scheme and a rough mesh resolution. Ambrosio used an Essentially Non-Oscillatory (ENO) scheme to study the shock-induced separated structures in symmetric corner flows [12]. Ambrosio and Goonko et al. [13-15] used the fifth-order weighted ENO scheme to capture the wave configurations induced by corner flows and compute the fluxes across cell faces. However, compared with the numerical and experimental research, the theoreti- 


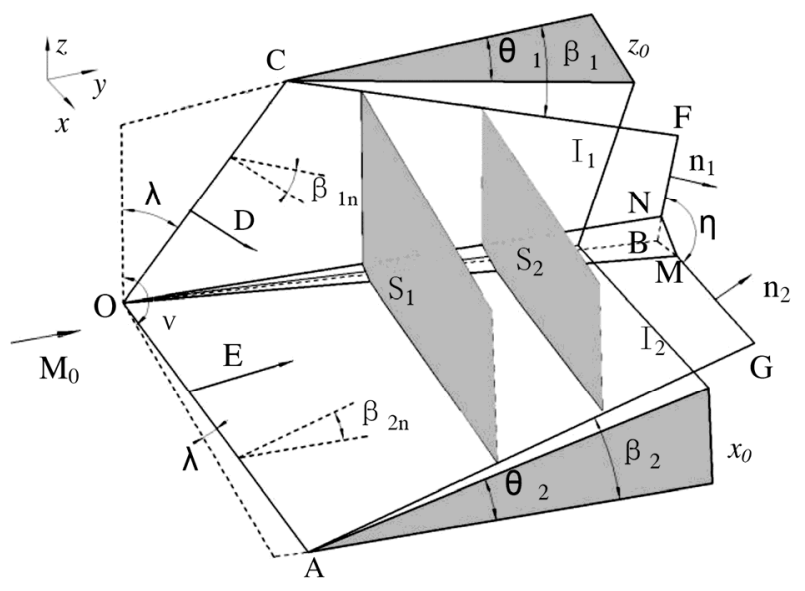

Figure 1 Schematic of a $M I$.

cal studies are far from satisfactory.

The earliest theory on shock reflection can be dated back to 1943 when Von Neumann built analytical approaches to solve regular reflection and Mach reflection, which were called the two-shock theory and three-shock theory, respectively $[18,19]$. Kawamura developed a method using polar coordinates $(p, \theta)$ to describe the shock-reflection phenomenon that is widely used today, where " $p$ " is the flow static pressure and " $\theta$ " is the flow deflection angle [20]. Ben-Dor systematically summarized the progress made in the two-dimensional (2D) shock-wave reflection phenomena and presented an analytical theory in his book [21]. In contrast to the well-established theory regarding the 2D shock reflection and interaction problem, there are few theoretical works on 3D shock reflection and interaction phenomena. Yang and Xiang [22-25] proposed a new theoretical method called "spatial-dimension reduction" to study the 3D shock/shock interaction induced by two intersecting wedges, and good agreements with numerical results were obtained.

In the present research, the 3D shock/shock interactions induced by two asymmetrical wedges are studied. The influences of asymmetrical wedge angles and sweep angles on flow field parameters and wave configurations are also considered. This paper is organized as follows: Section 2 presents the spatial-dimension reduction method and numerical approach in detail. Theoretical results and comparisons with numerical solutions are described in Section 3. A brief summary and conclusion are presented in Section 4.

\section{Theoretical analysis approach and numerical method}

\subsection{Theoretical analysis approach}

Figure 1 presents a classical model of two asymmetrical intersecting wedges, where $\lambda_{1}$ and $\lambda_{2}$ are the sweep angle, $\theta_{1}$ and $\theta_{2}$ are the wedge angle, and $v$ is the angle between the two bottom planes of wedges. For the inviscid super- sonic inflow $M_{0}$, two incident shock waves occur because of the compressional flows over the two wedges. The variables $\beta_{1}$ and $\beta_{2}$ are the shock angles on cross sections of wedges parallel to the direction of incoming flow, and $\beta_{1 n}$ and $\beta_{2 n}$ are the shock angles perpendicular to OA and OC, respectively. The two incident waves $I_{1}$ and $I_{2}$ interact with each other over the two intersecting wedges and several wave configurations may form, such as regular interaction $(R I)$, Mach interaction (MI), and weak-shock interaction (WSI). This paper focuses on the MI. A Mach stem surface occurs owing to the interaction of two incident shock waves. The main idea of the spatial-dimension reduction is converting the $3 \mathrm{D}$ steady problem to a $2 \mathrm{D}$ unsteady one so that we can use classical 2D theory combined with shock dynamics to solve the 3D shock/shock interaction. The main procedures are summarized as follows.

\subsubsection{Step 1: Geometrical transformations}

First, the relations between the 3D steady problem and 2D unsteady problem should be determined. Decomposing the 3D geometry into a $2 \mathrm{D}$ one is important to the problem transformation. For the inflow velocity $q$, it is not difficult to see that the intersecting line of the two incident waves $\mathrm{OB}$ is a unique direction, and the velocity $q_{n}$, which is parallel to it is identical throughout the whole flow field (see Figure 2). Thus, $\mathrm{OB}$ is defined as the characteristic direction, and the cross sections (such as $S_{1}$ and $S_{2}$ ) perpendicular to it are considered as the characteristic planes. After transforming the $3 \mathrm{D}$ steady problem into a $2 \mathrm{D}$ unsteady one, the problem can be regarded as two incident waves $M_{s 1}$ and $M_{s 2}$ moving along the virtual wall in a 2D plane (see Figures 2, 3(a), and 3(b)). The inflow velocity on characteristic planes is $q_{0}$, which passes through the incident waves and reflected waves in Figures 2 and 3(a). The virtual wall is the trajectory of the interacting point for $R I$ or a line perpendicular to the Mach stem, which was first defined by Xie [26].

The decomposed Mach number $M_{s 1}$ and $M_{s 2}$ can be calculated as follows:

$$
M_{s 1}=M_{0} \sin \beta_{1 n} \cos \lambda_{1 n}, M_{s 2}=M_{0} \sin \beta_{2 n} \cos \lambda_{2 n},
$$

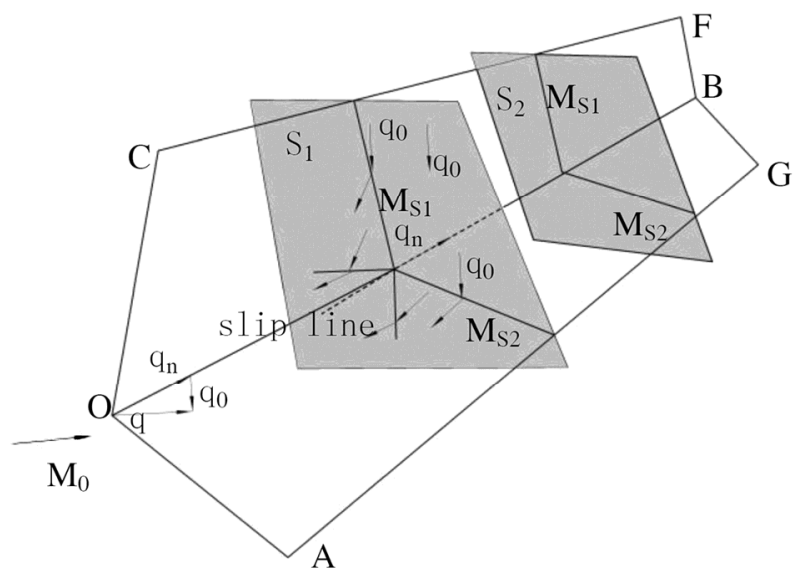

Figure 2 Schematic of "spatial-dimension reduction". 


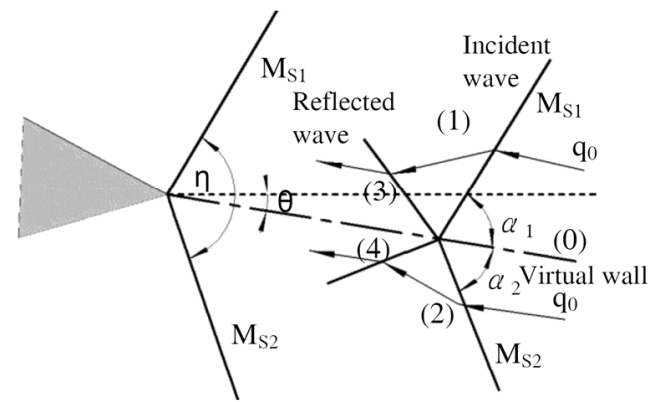

(a)

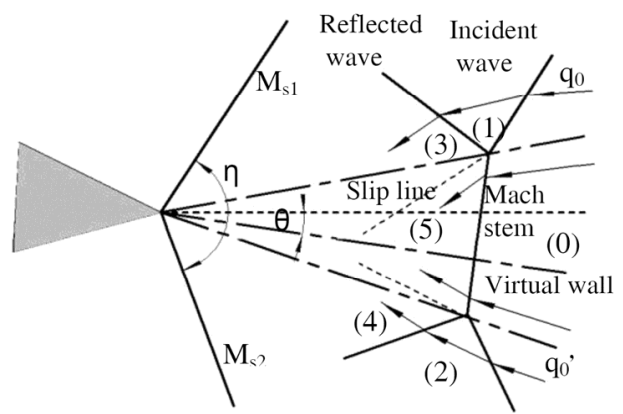

(b)

Figure 3 Schematic of a 2D moving shock/shock interaction. (a) $R I$; (b) MI.

where $\eta$ is the angle between $M_{s 1}$ and $M_{s 2}$ and can be obtained by the following equation:

$$
\cos \eta=\cos \left(\boldsymbol{n}_{1}, \boldsymbol{n}_{2}\right) \text {. }
$$

The vectors $\boldsymbol{n}_{1}$ and $\boldsymbol{n}_{2}$ on the cross sections can be expressed as

$$
\begin{aligned}
& \boldsymbol{n}_{1}=\boldsymbol{O} \boldsymbol{A} \times \boldsymbol{E} \\
& =\left(\cos \lambda_{1} \tan \beta_{1 n}-\cos \lambda_{1} \cot \lambda_{1} \tan \beta_{1 n}, \cos \lambda_{1}\right),
\end{aligned}
$$

$$
\boldsymbol{n}_{2}=\boldsymbol{D} \times \boldsymbol{O C}
$$

$=\left(\cot \lambda_{2} \sin v+\cos \lambda_{2} \cos v \tan \beta_{2 n}\right.$

$$
\left.-\csc \lambda_{2} \cos ^{2} \lambda_{2} \tan \beta_{2 n}, \cos \lambda_{2} \tan \beta_{2 n} \sin v-\cot \lambda_{2} \cos v\right) \text {, }
$$

where $\boldsymbol{E}$ is the vector in the shock surface $A O M$ normal to the leading edge of the bottom wedge, and $\boldsymbol{D}$ is the vector in shock surface $C O N$ normal to the leading edge of the lateral wedge.

\subsubsection{Step 2: Wave configuration determination}

The wave configuration is assumed as an $R I$ at first, and the 2D unsteady wave configuration can be observed in Figure 3(a). For the given inflow, the shock polar of the $R I$ can be determined using the following equation:

$$
\tan (\theta)=\frac{\xi-1}{\gamma M^{2}-(\xi-1)} \sqrt{\frac{\frac{2 \gamma}{\gamma+1}\left(M^{2}-1\right)-(\xi-1)}{\xi+\frac{\gamma-1}{\gamma+1}}},
$$

where $M$ is the decomposed Mach number in the direction of the reflection point, $\gamma$ is 1.4 for an ideal gas, and $\xi$ is the ratio of the pressure behind $M_{s 1}$ and $M_{s 2}$.

The determinations of the wave configuration according to the assumed shock polar are as follows (Figure 4(a)).

1) The reflected polars $R_{1}$ and $R_{2}$ intersect with each other at two points, and an $R I$ occurs.

2) The reflected polars $R_{1}$ and $R_{2}$ do not intersect with each other but intersect with incident polars $I_{1}$ and $I_{2}$, respectively, and an $M I$ occurs.

3) The reflected polars $R_{1}$ and $R_{2}$ are totally inside the two incident polars $I_{1}$ and $I_{2}$, respectively, and a WSI occurs.

\subsubsection{Step 3: Solutions to the 2D unsteady problem}

For the $R I$, the solution to the flow field can be obtained by shock polar analysis. The pressure behind two reflected waves is the value for the lower intersecting point $O_{1}$.

For the $M I$, the Mach stem gets longer with the propagation of two incident waves $M_{s 1}$ and $M_{s 2}$, which can be viewed as the characteristic plane propagating along the characteristic direction. The angle between the virtual wall and the horizontal line $\theta_{v}$, and the Mach number behind the Mach stem $M_{m}$, can be calculated using the following equations [26]:

$$
\tan \theta_{v}=\left(\frac{M_{m}}{M_{s 2}}\right) \frac{\left[1-\left(\frac{M_{s 2}}{M_{m}}\right)^{2}\right]^{\frac{1}{2}}\left\{1-\left[\frac{f\left(M_{m}\right)}{f\left(M_{s 2}\right)}\right]^{\frac{1}{2}}\right\}}{1+\frac{f\left(M_{m}\right) M_{m}}{f\left(M_{s 2}\right) M_{s 2}}},
$$

$$
\begin{aligned}
& \tan \left(\pi-\eta-\theta_{v}\right) \\
& =\left(\frac{M_{m}}{M_{s 1}}\right) \frac{\left[1-\left(\frac{M_{s 1}}{M_{m}}\right)^{2}\right]^{\frac{1}{2}}\left\{1-\left[\frac{f\left(M_{m}\right)}{f\left(M_{s 1}\right)}\right]^{\frac{1}{2}}\right\}}{1+\frac{f\left(M_{m}\right) M_{m}}{f\left(M_{s 1}\right) M_{s 1}}},
\end{aligned}
$$

where $f(M)$ is a function in terms of Mach number $M$, and its expression is

$$
f(M)=\exp \left[-\int \frac{2 M \mathrm{~d} M}{\left(M^{2}-1\right) K(M)}\right],
$$

$K(M)$ is a slowly varying function and its expression is

$$
K(M)=2\left(2 \mu+1+M^{-2}\right)^{-1}\left(1+\frac{2}{\gamma+1} \frac{1-\mu^{2}}{\mu}\right)^{-1},
$$

where $\mu=\left[\frac{(\gamma-1) M^{2}+2}{2 \gamma M^{2}-(\gamma-1)}\right]^{\frac{1}{2}}$, which represents a function of the Mach number of a moving shock relative to the 

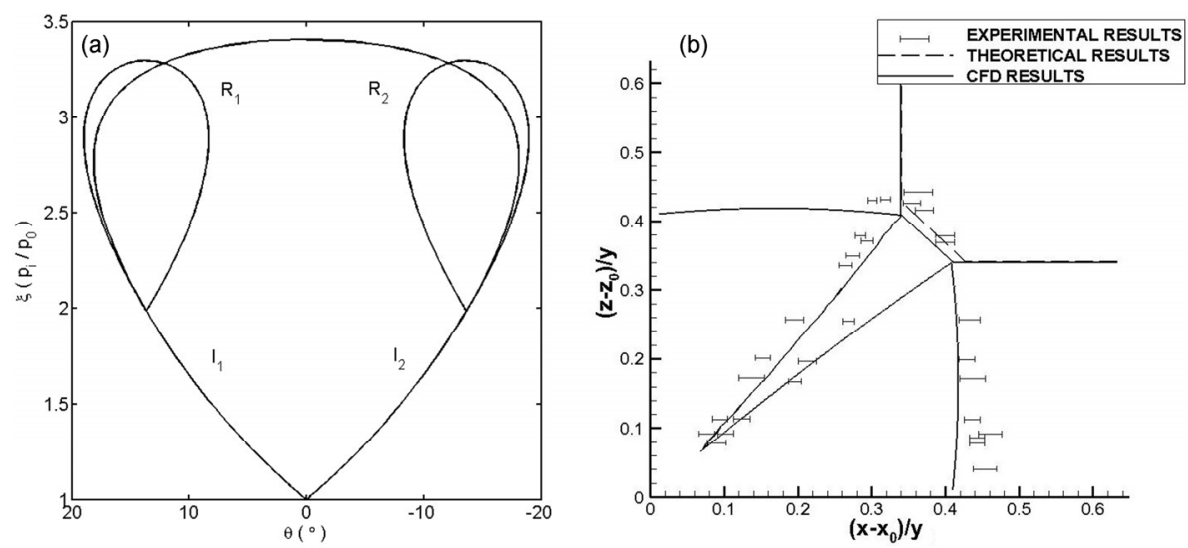

Figure 4 Comparison with experimental and numerical results: (a) shock polar analysis on characteristic plane; (b) wave configuration of an MI.

flow field ahead of it.

\subsubsection{Step 4: Solutions to the 3D steady problem}

Finally, the values of all zones should be calculated for such 3D problems. The state parameters, such as the temperature, pressure, density, and total pressure recovery coefficient, are identical to those of the $2 \mathrm{D}$ unsteady solutions. The vector parameters, such as the velocities and Mach number, should be composed with the decomposed vectors along OB in Figures 1 and 2. Therefore, all the flow-field parameters can be solved.

\subsection{Numerical method}

The governing equation is the following 3D Euler equation:

$$
\frac{\partial U}{\partial t}+\frac{\partial F}{\partial x}+\frac{\partial G}{\partial y}+\frac{\partial H}{\partial z}=0
$$

where $U, F$, and $G$ are the state variables and fluxes in the $x$-, $y$-, $z$ - directions, respectively, and their expressions are as follows:

$$
\begin{aligned}
U & =\left(\begin{array}{l}
\rho \\
\rho u \\
\rho v \\
\rho w \\
\rho E
\end{array}\right), F=\left(\begin{array}{l}
\rho u \\
\rho u^{2}+p \\
\rho u v \\
\rho u w \\
(\rho E+p) u
\end{array}\right), \\
G & =\left(\begin{array}{l}
\rho v \\
\rho u v \\
\rho v^{2}+p \\
\rho v w \\
(\rho E+p) v
\end{array}\right), H=\left(\begin{array}{l}
\rho w \\
\rho u w \\
\rho v w \\
\rho w^{2}+p \\
(\rho E+p) w
\end{array}\right) .
\end{aligned}
$$

For the ideal gas, the total internal energy per unit mass $\boldsymbol{E}$ is

$$
\boldsymbol{E}=\frac{p}{(\gamma-1) \rho}+\frac{1}{2}\left(u^{2}+v^{2}+w^{2}\right)
$$

The second-order NND scheme is applied for the convective terms $F, G$, and $H$ [27]. The discretization of the governing equation is as follows:

$$
\begin{aligned}
& \left(\frac{\partial U}{\partial t}\right)_{i, j, k}^{n}+\frac{1}{\Delta x}\left(F_{i+\frac{1}{2}, j, k}^{n}-F_{i-\frac{1}{2}, j, k}^{n}\right) \\
& +\frac{1}{\Delta y}\left(G_{i, j+\frac{1}{2}, k}^{n}-G_{i, j-\frac{1}{2}, k}^{n}\right)+\frac{1}{\Delta z}\left(H_{i, j, k+\frac{1}{2}}^{n}-H_{i, j, k-\frac{1}{2}}^{n}\right)=0 .
\end{aligned}
$$

The computational mesh uses the orthogonalized uniform structured mesh, and the mesh number is $120 \times 200 \times 200$ in the $x, y$, and $z$ directions. The MPI Parallel Program is used in the code. Mesh independence tests were performed to ensure that all the results produced were independent of the type of mesh chosen for the numerical simulations. The inlet of the computational zone was given by a fixed inflow, the far-field selected the non-reflecting boundary condition, and the walls selected the solid slipping condition. The computations were conducted on an 8-core computer (Dell, USA).

\section{Results and discussion}

The analytical method is validated by comparing the theoretical result with the numerical and experimental results. Figure 4(a) presents the shock polar analysis on the characteristic plane using this new analytical method. The selected inflow and geometrical parameters are $M_{0}=3, \theta_{1}=\theta_{2}=9.5^{\circ}$, $\lambda_{1}=\lambda_{2}=0^{\circ}, \nu=90^{\circ}$. The horizontal axis $\theta$ is the flow deflection angle on the characteristic plane, and the vertical axis $\xi$ is the static-pressure ratio. The reflected polars $R_{1}$ and $R_{2}$ intersect with the incident polars $I_{1}$ and $I_{2}$, respectively, at two points and an $M I$ occurs, which has been verified by experimental and numerical results (see Figure 4(b)). As shown in Figure 4(b), the $x$ and $z$ coordinates are scaled with $y$ so that they become conical, self-similar variables. $x_{0}$ and $z_{0}$ are the height of bottom wedge and upper wedge on 
cross section respectively, which have been shown in Figure 1. The error bars show the experimental results of West in 1972 [4], the solid lines show the numerical results, and the dashed lines show the theoretical results. The analytical results agree well with the experimental and numerical results. The theoretical solution for the Mach number behind the Mach stem is $\sim 1.84$, which fits the numerical result $M_{m}$ $=1.82$ well.

In the following sections, the asymmetry of the flow characteristics and wave configurations induced by the wedge angle and sweep angle are discussed and analyzed thoroughly. Additionally, the theoretical results are compared with the numerical results, revealing good agreement.

\subsection{Asymmetry of the wedge angle}

To explore the effects of asymmetry induced by the wedge angle on wave configuration and parameters of the flow field, the wedge angle on one side is fixed at $12.2^{\circ}$, and that on the other side varies from $3.5^{\circ}$ to $15^{\circ}$. The inflow Mach number is 3.17 , and the sweep angle and dihedral angle are selected as $0^{\circ}$ and $90^{\circ}$, respectively. Figure 5 shows the shock-polar with different wedge angles and indicates the wave configurations are $M I$ in all cases. As the wedge angle increases on one side, the incident polar $I_{i}$ and $I_{i}{ }^{\prime}$ on both sides become larger and higher, the reflected polar $R_{i}^{\prime}$ on the side of varying wedge angle increases, and the reflected polar $R_{i}$ on the side of fixed wedge angle decreases. Figure 6 presents the numerical solutions to study the effects of the asymmetrical wedge angle on wave configurations. As the wedge angle on one side increases, the incident waves $1_{i}$ and $1_{i}{ }^{\prime}$, the reflected waves $2_{i}$ and $2_{i}{ }^{\prime}$, and the Mach stem $3_{i}$, get further from the corner points $O_{i}$. The dashed lines that originate from $O_{i}$ are perpendicular to the Mach stem or the extension line of the Mach stem. If the foot of the perpendicular is on the extension line, the $M I$ is an inversed $M I$. It should be noted that when the foot of the perpendicular is on the extension line, the reflected polar $R_{1}$ intersects with the incident polar $I_{1}{ }^{\prime}$ on the negative side of the $\theta$ axis (Figure 5, solid line at $\theta_{2}=3.5^{\circ}$ ). The Mach stem gets longer as the wedge angle increases and becomes inversed if the wedge angles on both sides have a larger discrepancy.

Figure 7 displays the theoretical solutions in the vicinity of the Mach stem, which includes the total pressure recovery coefficient $\theta_{p}$, Mach number behind the Mach stem $M_{m}$, pressure $P$, temperature $T$, and density $\rho$. The horizontal axis $\theta_{2}$ represents the varying wedge angle in one side. The initial inflow parameters are non-dimensionalized as unit one in zone zero, $P_{0}=1, \rho_{0}=1, T_{0}=1, \theta_{p 0}=1$. As the wedge angle $\theta_{2}$ increases, the pressure, density, and temperature increase monotonously in zones (2)-(5) and remain constant in zone (1). As zones (3) to (5) are divided by two slip faces, they have the same pressure. The $P_{2}$ on the side of varying wedge angle behind the incident wave is smaller than $P_{1}$ on the side of fixed wedge angle when $\theta_{2}<\theta_{1}$; in contrast, $P_{2}>P_{1}$ at $\theta_{2}>\theta_{1}$ (Figure $7(\mathrm{c})$ ). As indicated in Figure 7(d) and (e), the temperature in zone (5) behind the Mach stem is higher than that in zones (3) and (4), whereas the density in zone (5) is lower than that in zones (3) and

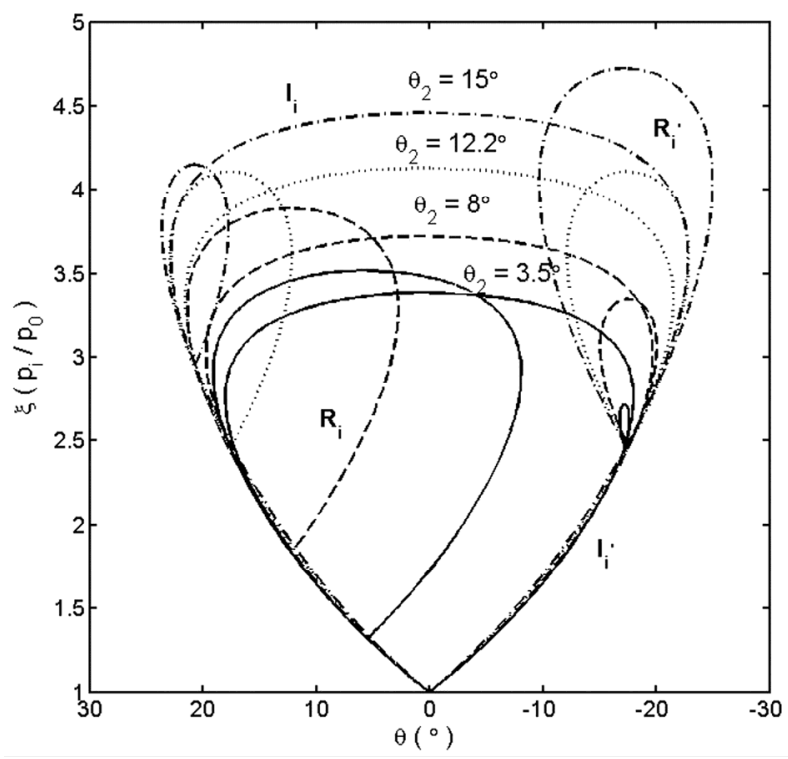

Figure 5 Shock-polar for different wedge angles. $I_{i}$ - incident polar on the side of the fixed wedge, $I_{i}{ }^{\prime}$ - incident polar on the side of the varying wedge, $R_{i}$ - reflected polar on the side of the fixed wedge, $R_{i}{ }^{\prime}-$ reflected polar on the side of the varying wedge $\left(i=1\left(\theta_{2}=3.5^{\circ}\right), 2\left(\theta_{2}=8^{\circ}\right), 3\left(\theta_{2}=\right.\right.$ $\left.\left.12.2^{\circ}\right), 4\left(\theta_{2}=15^{\circ}\right)\right)$.

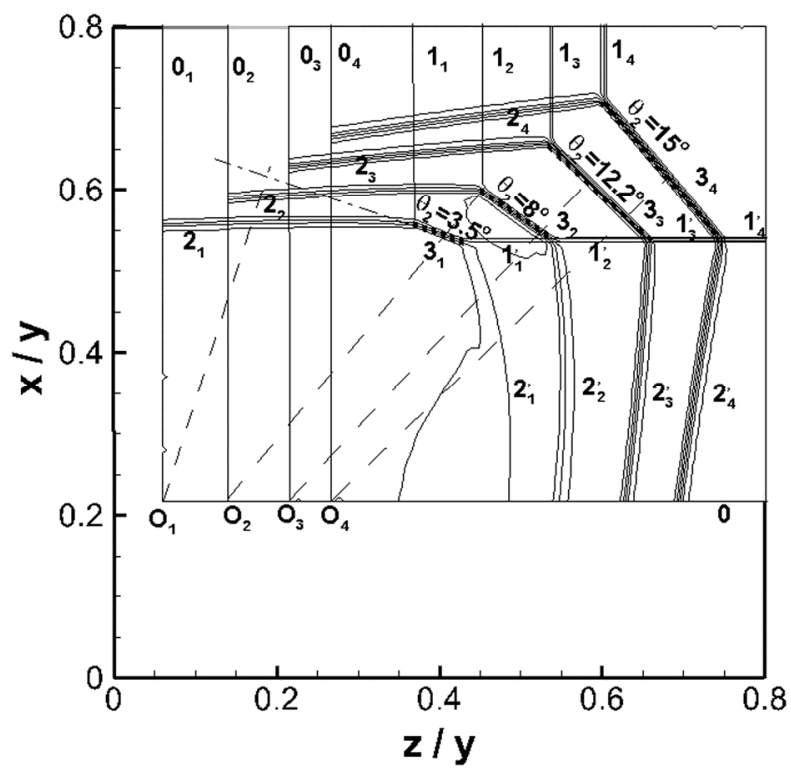

Figure 6 Numerical results for different wedge angles. 0 - wall boundary on the side of the fixed wedge, $0_{i}{ }^{\prime}$ - wall boundary on the side of the varying wedge, $1_{i}$ - incident wave on the side of the varying wedge, $1_{i}{ }^{\prime}$ - incident wave on the side of the fixed wedge, $2_{i}{ }^{\prime}$ - reflected wave on the side of the fixed wedge, $2_{i}{ }^{\prime}$ - reflected wave on the side of the varying wedge, $3_{i}$ - Mach stem, $O_{i}-$ corner points $\left(i=1\left(\theta_{2}=3.5^{\circ}\right), 2\left(\theta_{2}=8^{\circ}\right), 3\left(\theta_{2}=12.2^{\circ}\right)\right.$, $\left.4\left(\theta_{2}=15^{\circ}\right)\right)$. 

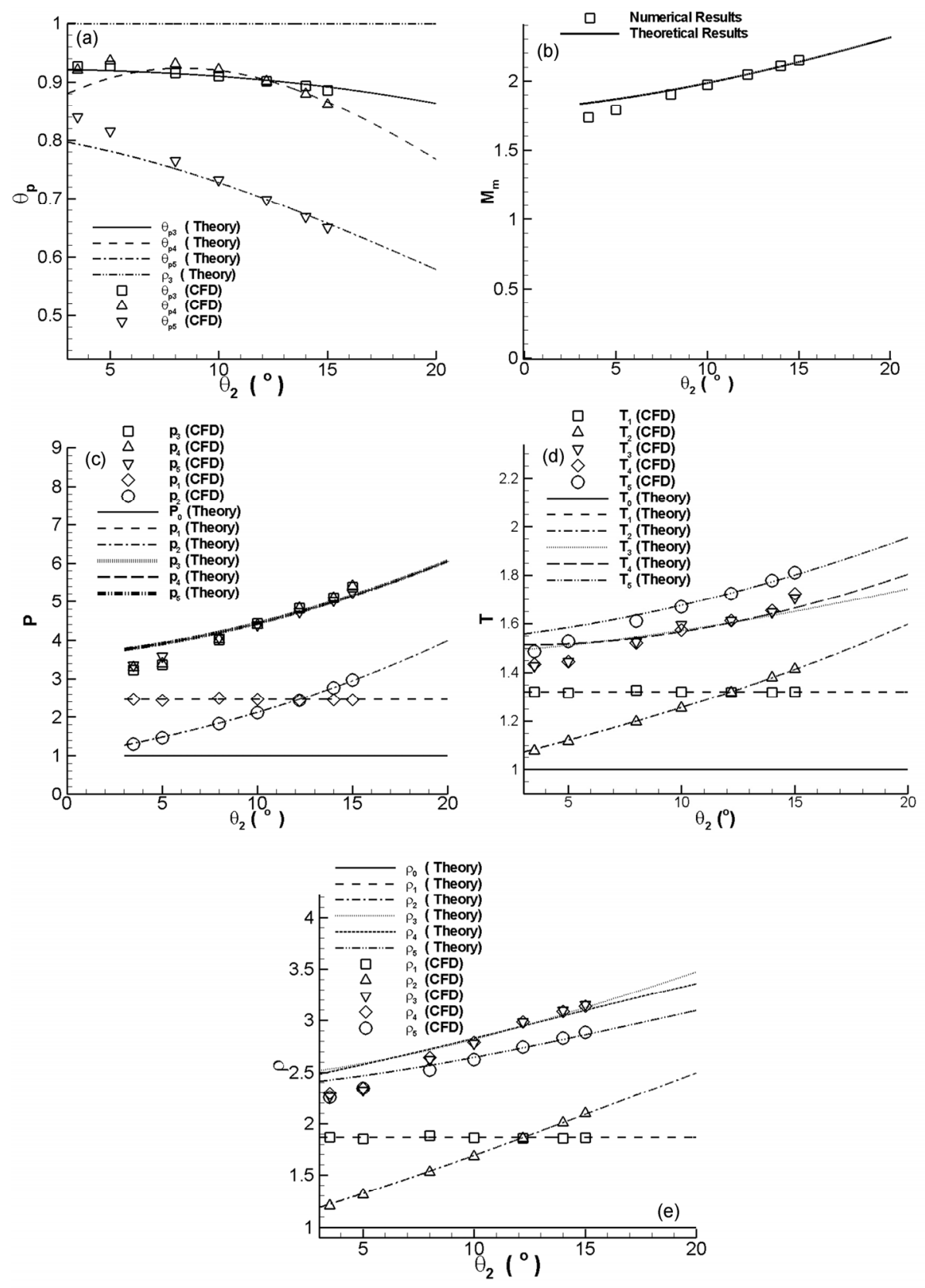

Figure 7 Flow-field parameter variations with respect to the wedge angle. (a) Total pressure recovery coefficient; (b) Mach number behind the Mach stem; (c) pressure; (d) temperature; (e) density.

(4). When $\theta_{2}<\theta_{1}$, the temperature and density are almost the same between zones (3) and (4). When $\theta_{2}>\theta_{1}$, the temperature in zone (3) behind the reflected wave is lower than that in zone (4), and the density in zone (3) is higher than that in zone (4). Figure 7(a) and (b) present the variations of total pressure recovery coefficient and Mach number behind the Mach stem with the increasing wedge angle on one side. The increasing wedge angle on one side induces the increase of the Mach number behind the Mach stem and the decrease of total pressure recovery coefficient in zones (3) to (5). As $\theta_{2}$ increases, $\theta_{p 3}$ on the side of fixed wedge angle varies slightly, and $\theta_{p 4}$ on the side of varying wedge angle first increases, then decreases. The total pressure loss in zone (5) behind the Mach stem is more serious than that in zones (3) and (4) behind the reflected waves because the entropy production passing through the Mach stem is larger than that passing through the incident wave and the reflected wave.

\subsection{Asymmetry of the sweep angle}

The studies on effects of the sweep angle are conducted by fixing one sweep angle $\lambda_{2}$ at $10^{\circ}$ and varying another sweep angle from $0^{\circ}$ to $30^{\circ}$. The inflow Mach number, wedge an- 
gle, and dihedral angle are $4^{\circ}, 10^{\circ}, 90^{\circ}$ respectively. As shown in Figure 8, the increase of sweep angle on one side make the incident polar and reflected polar small. However, the asymmetry induced by the sweep angle has little influence on two incident polars $I_{i}$ and $I_{i}^{\prime}$ as $\lambda_{1}$ varies from $0^{\circ}$ to $20^{\circ}$. When $\lambda_{1}$ increases to $30^{\circ}, I_{4}{ }^{\prime}$ is obviously smaller than $I_{4}$, owing to the large discrepancy between the two sweep angles.

The shock polar analysis shown in Figure 8 indicates that the wave configurations are $M I$ for the aforementioned cases, which has been validated by numerical simulations in Figure 9. As the sweep angle on one side increases, the incident waves on the side of varying sweep angle move closer to the wall boundary, and the Mach stem gets closer to the corner point $\mathrm{O}$ and becomes longer. For the reflected wave, the increasing sweep angle on one side makes $2_{i}{ }^{\prime}$ farther from the corner and $2_{i}$ closer to the corner. Compared with the effects of the wedge angle, the influence of the sweep angle on the wave configuration is not obvious (see Figures 6 and 9).

Figure 10 presents the theoretical solutions for the flow field in the vicinity of the triple point, i.e., the intersecting point of the incident wave, reflected wave, and Mach stem. Compared with the wedge angle, the influence of sweep angle on variations of the pressure, temperature, density, Mach number behind the Mach stem, and total pressure recovery coefficient exhibits the opposite trend. As the sweep angle $\lambda_{1}$ on one side increases, the Mach number behind Mach stem, pressure, temperature, and density in zones (3) to (5) decrease slightly, while the total pressure recovery coefficient in these zones increases slowly. Unlike the asymmetry of the wedge angle, the asymmetry of the

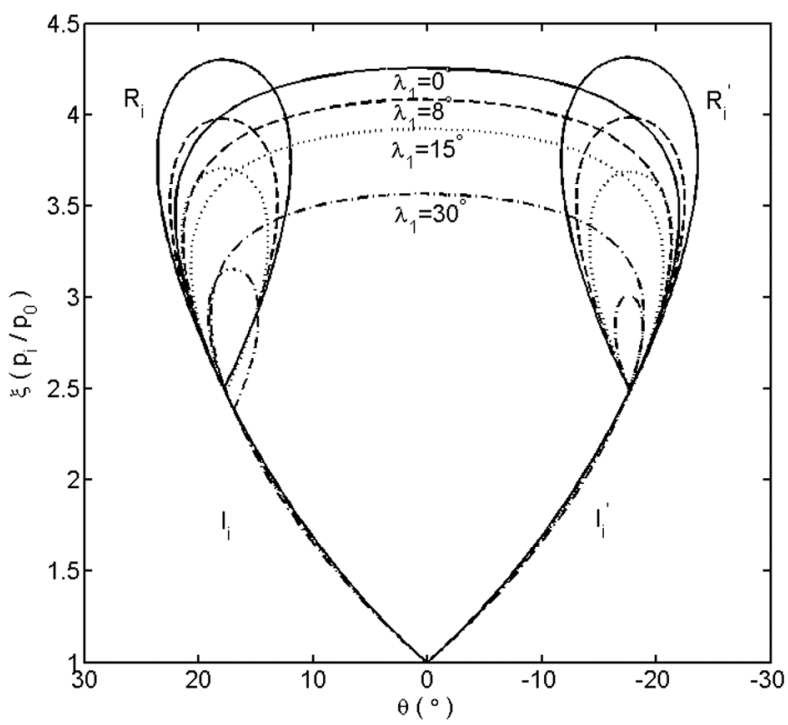

Figure 8 Shock-polar with different sweep angles. $I_{i}$ - incident polar on the side of fixed wedge, $I_{i}{ }^{\prime}$ - incident polar on the side of varying wedge, $R_{i}{ }^{\prime}$ - reflected polar on the side of fixed wedge, $R_{i}{ }^{\prime}-$ reflected polar on the side of varying wedge $\left(i=1\left(\lambda_{1}=0^{\circ}\right), 2\left(\lambda_{1}=8^{\circ}\right), 3\left(\lambda_{1}=15^{\circ}\right), 4\left(\lambda_{1}=\right.\right.$ $\left.30^{\circ}\right)$ ).

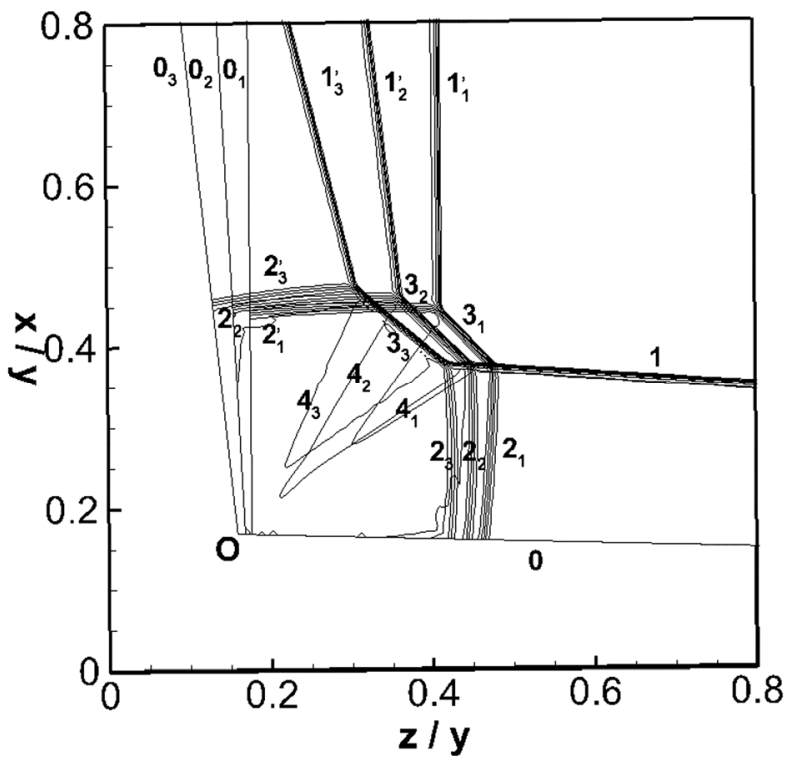

Figure 9 Numerical results for different wedge angles. 0 - wall boundary on the side of fixed sweep angle, $0_{i}$ - wall boundary on the side of varying sweep angle, $1_{i}{ }^{\prime}-$ incident wave on the side of varying sweep angle, $1-$ incident wave on the side of fixed sweep angle, $2_{i}$ - reflected wave on the side of fixed sweep angle, $2{ }_{i}{ }^{\prime}-$ reflected wave on the side of varying sweep angle, $3_{i}$ - Mach stem, $O$ - corner point $\left(i=1\left(\lambda_{1}=0^{\circ}\right), 2\left(\lambda_{1}=15^{\circ}\right), 3\left(\lambda_{1}\right.\right.$ $\left.=30^{\circ}\right)$ ).

sweep angle does not cause a difference in the flow parameters behind the two incident waves in zones (1) and (2) and the two reflected waves in zones (3) and (4) (see Figures $10(c)-(e))$. The effects of the asymmetry of the sweep angle on one side on the flow-field parameters are negligible and do not cause the distinct asymmetry of the flow field.

\section{Conclusion}

A method called spatial-dimension reduction is used to study the 3D asymmetrical shock/shock interaction induced by two intersecting wedges. First, the theoretical method is introduced in detail. With this analytical approach, not only can the wave configuration be determined, but also the solutions to flow field in the vicinity of the triple point can be obtained. Secondly, numerical simulations are conducted to verify the theoretical results by solving 3D inviscid Euler equations with a classical NND scheme. Finally, the effects of the asymmetry of the wedge angle and sweep angle on the wave configurations and flow-field parameters are discussed thoroughly. The results can be summarized as follows.

1) The asymmetrical shock/shock interaction can be solved by the spatial-dimension reduction method, including the wave configuration and flow-field parameters, and good agreements are obtained with the numerical results.

2) The asymmetry of wedge angle induces the clear asymmetry of the flow field behind the two incident waves 

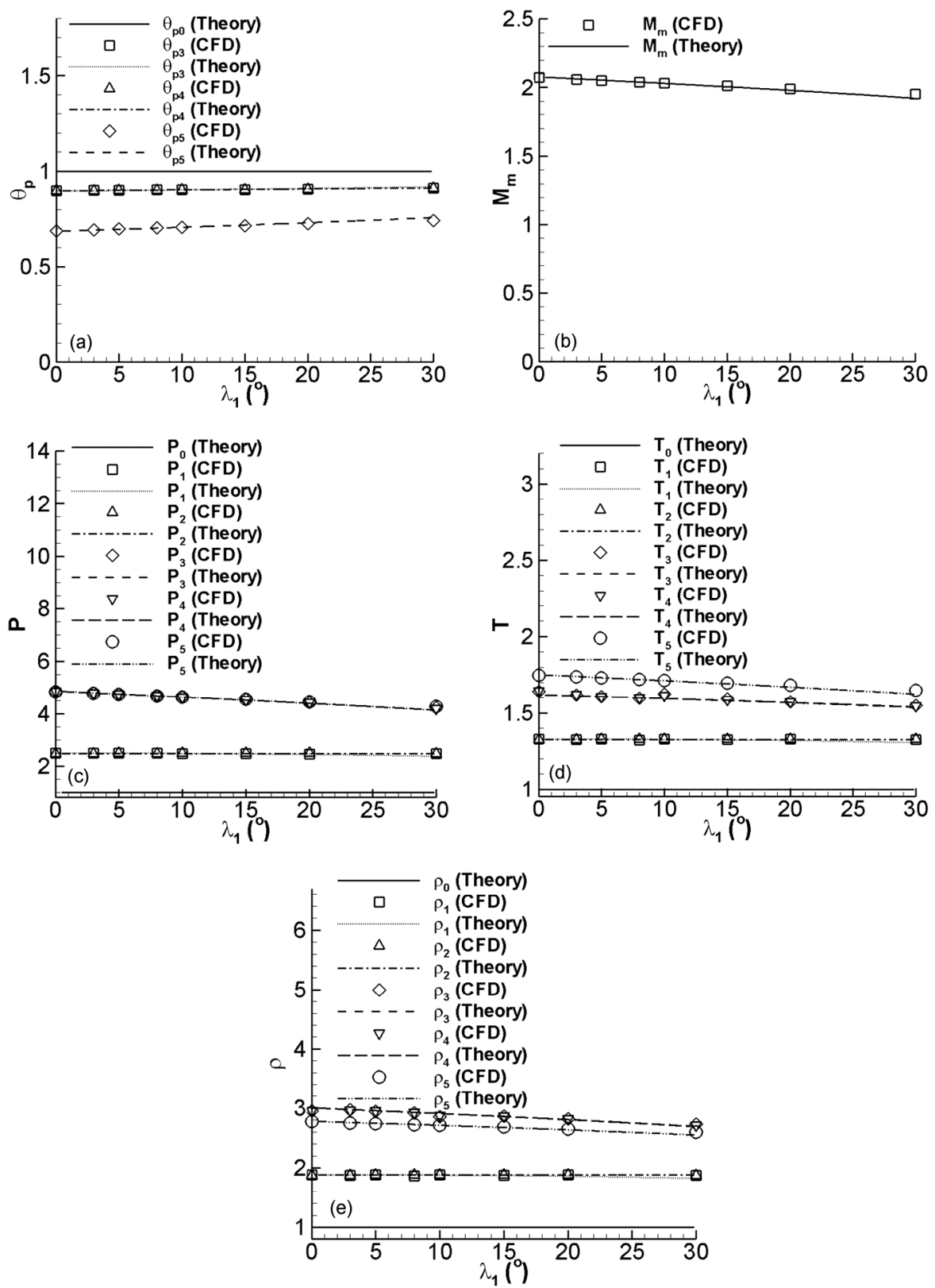

Figure 10 Flow field parameter variations with respect to wedge angle. (a) Total pressure recovery coefficient; (b) Mach number behind the Mach stem; (c) pressure; (d) temperature; (e) density.

and reflected waves, while the asymmetry of the sweep angle does not result in the obvious asymmetry of the flow field in zones (1)-(4).

3) The Mach number behind the Mach stem increases sharply as the wedge angle on one side increases and decreases slightly with the increasing sweep angle on one side. Furthermore, the total pressure loss behind the Mach stem is more serious with the increasing wedge angle than with the increasing sweep angle.

4) The influence of varying the wedge angle on the wave configurations is significant, whereas the effect of varying sweep angle on the wave configurations is negligible.
This work was supported by the National Natural Science Foundation of China (Grant No. 11372333). The authors would like to thank Pro. Luo and Teng for their valuable assistance.

1 Charwat A F, Redekeopp L G. Supersonic interference flow along the corner of intersecting wedges. AIAA J, 1967, 5: 480-488

2 Cresci R J, Lin T C, Nardo C T, et al. Hypersonic interaction along a rectangular corner. AIAA J, 1969, 7: 2241-2246

3 Watson R, Weinstein L. A study of hypersonic corner flow interactions. AIAA J, 1971, 9: 1280-1286

4 West J E, Korkegi R H. Interaction of the corner of intersecting wedges at a Mach number of 3 and high Reynolds numbers. AIAA J, 1972, 10: 652-656

5 Stainback P C, Weinstein L M. Aerodynamic Heating in the Vicinity of Corners at Hypersonic Speeds. Washington D. C.: Natl Aero- 
nautics Space Administration, 1967

6 Korkegi R H. Survey of viscous interactions associated with high Mach number flight. AIAA J, 1971, 9: 771-784

7 Venkateswaran S, Witte D W, Hunt L R. Aerothermal study in an axial compression corner with shock impingement at Mach 6. AIAA Paper No. 91-0527, 1991

8 Rubin S G. Incompressible flow along a corner. J Fluid Mech, 1966, 26: $97-110$

9 Kutler P. Supersonic flow in the corner formed by two intersecting wedges. AIAA J, 1974, 12: 577-578

10 Shang J S, Hankey W L, Petty J S. Three-dimensional supersonic interacting turbulent flow along a corner. AIAA J, 1979, 17: 706-713

11 Marconi F. Supersonic, inviscid, conical corner flowfields. AIAA J, 1980, 18: 78-84

12 Ambrosio D, Marsilio R. Shock-induced separated structures in symmetric corner flows. NASA Langley Research Center. Institute for Computer Applications in Science and Engineering [ICASE], ICASE Report, 1995. 79-95

13 Goonko Y P, Kudryavtsev A N, Chpoun A. 3D interaction of shock waves in corner flow. Berlin: Shock Waves, Springer Berlin Heidelberg, 2005. 437-442

14 Goonko Y P, Kudryavtsev A N, Rakhimov R D. Supersonic inviscid corner flows with regular and irregular shock interaction. Fluid Dyn, 2004, 39: 304-318

15 Goonko Y P, Kudryavtsev A N. Numerical modelling of free interaction of shock waves in corner flows. Thermophys Aeromechanics, 2006, 13: 221-237

16 Ning J G, Wang X, Ma T B, et al. Numerical simulation of shock wave interaction with a deformable particle based on the pseudo arc-length method. Sci China Tech Sci, 2015, 58: 848-857
17 Hongzhi L I, Baochun F A N, Jihui G, et al. Shock wave interaction with surface of combustible dust layers. Sci China Ser E-Tech Sci, 1996, 39: 449-460

18 Von Neumann J. Refraction, intersection and reflection of shock waves. NAVORD Rep, 1945, 1: 203-245

19 von Neumann J. Oblique Reflection of Shock Waves, Collected Work of Von Neumann, John. Oxford: Pergamon Press, 1996. 145: 44-46

20 Kawamura R, Saito H. Reflection of shock waves-1 Pseudo-stationary case. J Phys Society Japan, 1956, 11: 584-592

21 Ben-Dor G. Shock Wave Reflection Phenomena. Berlin: Springer, 2007

22 Yang Y. The investigations on complex flow of three dimensional shock/shock interaction (in Chinese). Dissertation of Doctor Degree. Beijing: Chinese Academy of Sciences, 2012

23 Xiang G, Wang C, Teng H, et al. Study on Mach stems induced by interaction of planar shock waves on two intersecting wedges. Acta Mechanica Sinica, 2015, doi: 10.1007/s10409-015-0498-2

24 Yang Y, Wang C, Jiang Z L. Analytical and numerical investigations of the reflection of asymmetric nonstationary shock waves. Shock Waves, 2012, 22: 435-449

25 Xiang G X, Wang C, Teng $\mathrm{H} \mathrm{H}$, et al. Investigations of three- dimensional shock/shock interactions over symmetrical intersecting wedges. AIAA J, 2016, doi:10.2514/1.J054672

26 Xie P, Han Z Y, Takayama K. A study of the interaction between two triple points. Shock Waves, 2005, 14: 29-36

27 Zhang H X. A dissipative difference scheme of non-oscillatory, nofree parameters (in Chinese). Acta Aerodynamica Sinica, 1988, 6: 143-165 